\title{
PARITY OF THE COEFFICIENTS OF KLEIN'S $j$-FUNCTION
}

\author{
CLAUDIA ALFES
}

(Communicated by Ken Ono)

\begin{abstract}
Klein's $j$-function is one of the most fundamental modular functions in number theory. However, not much is known about the parity of its coefficients. It is believed that the odd coefficients are supported on "one half" of the arithmetic progression $n \equiv 7(\bmod 8)$. Following a strategy first employed by Ono for the partition function, we use twisted Borcherds products and results on the nilpotency of the Hecke algebra to obtain new results on the distribution of parity for the coefficients of $j(z)$.
\end{abstract}

\section{INTRODUCTION AND STATEMENT OF RESULTS}

$j(z):=\frac{E_{4}(z)^{3}}{\Delta(z)}=\frac{\left(1+240 \sum_{n=1}^{\infty} \frac{n^{3} q^{n}}{1-q^{n}}\right)^{3}}{q \prod_{n=1}^{\infty}\left(1-q^{n}\right)^{24}}=q^{-1}+744+196884 q+21493760 q^{2} \cdots$,

where $q:=e^{2 \pi i z}$ and $z \in \mathbb{H}$. Its properties and connections to number theory, elliptic curves, class field theory and moonshine are well known.

However, surprisingly little is known about the behavior of the coefficients of $j(z)$ modulo a prime. Perhaps the most famous congruence is due to Lehner [5]. For primes $p \leq 11$, he showed that

$$
j(z) \mid U(p) \equiv 744 \quad(\bmod p),
$$

where $j(z) \mid U(p)=\sum_{n=-1}^{\infty} c(n) q^{n / p}$ and the summation is only carried out over $n$ 's that are divisible by $p$. Lehner [6], 7] and Kolberg [4] later expanded on such congruences. Serre [11] showed that congruences of the $U(p)$-type do not hold for primes $p>11$. Ahlgren and Ono [1] generalized Lehner's and Serre's result to all weakly holomorphic modular forms (see Theorems 2 and 4 of [1]).

Here we address the parity of the coefficients of $j(z):=\sum_{n=-1}^{\infty} c(n) q^{n}$. Since we have $E_{4}(z) \equiv 1(\bmod 2)$, (1.1) easily gives

$$
j(z) \equiv \frac{1}{q \prod_{n=1}^{\infty}\left(1-q^{8 n}\right)^{3}} \equiv q^{-1}+q^{7}+q^{15}+q^{31}+\cdots \quad(\bmod 2) .
$$

Therefore, the odd coefficients are supported on the arithmetic progression $n \equiv 7$ $(\bmod 8)$. A short calculation reveals that $6.275 \%$ of the first $1,000,000$ coefficients of $j(z)$ are odd. More generally, it is conjectured by Ono that this holds for $1 / 16$ of the $c(n)$. Note that $1 / 16=0.0625$.

Received by the editors June 10, 2011.

2010 Mathematics Subject Classification. Primary 11F03, 11F30, 11 F33.

The author was supported by the German Academic Exchange Service. 
Here we offer new results on the distribution of parity of the coefficients of $j(z)$. To be more precise, we let $\Delta=4 \cdot \Delta^{\prime}$ be a positive fundamental discriminant (i.e. $\Delta^{\prime} \equiv 2,3(\bmod 4)$ and $\Delta^{\prime}$ square-free $)$. Then we define the following generating functions:

$$
\tilde{J}(\Delta ; z):=\sum_{\substack{m=1 \\ \operatorname{gcd}(m, 2)=1}}^{\infty} c\left(\Delta^{\prime} m^{2}\right) \sum_{\substack{n=1 \\ \operatorname{gcd}(n, \Delta)=1}}^{\infty} q^{n m} .
$$

Theorem 1.1. We have that $\tilde{J}(\Delta ; z)$ is congruent modulo 2 to a weight 2 meromorphic modular form on $\Gamma:=S L_{2}(\mathbb{Z})$ with integer coefficients whose poles are simple and are supported on CM points of discriminant $-4 \Delta$.

Using the theory of modular forms modulo 2, we use this result to obtain the following result on the distribution of parity for the coefficients of $j(z)$.

Theorem 1.2. For a prime $\ell \equiv 7(\bmod 8)$, there are infinitely many odd $m$ for which $c\left(\ell m^{2}\right)$ is even. Moreover, the first such $m$ is bounded by $(\ell+1)(24 H(-16 \ell)+$ 4).

Remark. This theorem follows from the more general Theorems 1.3 and 1.4 below using bounds for the Hurwitz-Kronecker class numbers $H(-4 \Delta)$, the class number of quadratic forms of discriminant $-4 \Delta$, where each class $C$ is counted with multiplicity $1 / \operatorname{Aut}(C)$, and indices of standard congruence subgroups.

Remark. It follows from the proof of Theorem 1.2 that for each $\ell$ there is an odd $m$ satisfying

$$
m \leq 6 H(-16 \ell)+1 \ll \ell^{3 / 2} \log (\ell)
$$

such that $c\left(\ell m^{2}\right)$ is even. The strong form of Dirichlet's theorem on primes in arithmetic progressions implies the following estimate:

$$
\#\{N \leq X: c(N) \text { is even }\} \gg \frac{X^{1 / 4}}{\log (X)} .
$$

Theorem 1.3. The following are true:

(1) There are infinitely many odd $m$ for which $c\left(\Delta^{\prime} m^{2}\right)$ is even if there is at least one such $m$. Moreover, if there are any $m$, then the first one is bounded by $\frac{12 H(-4 \Delta)+2}{12}\left[\Gamma: \Gamma_{0}\left(4 \Delta^{\prime}\right)\right]$.

(2) There are infinitely many odd $m$ for which $c\left(\Delta^{\prime} m^{2}\right)$ is odd if there is at least one such $m$. Moreover, if there are any $m$, then the first one is bounded by $\frac{1}{12}(12 H(-4 \Delta)+2)$.

Theorem 1.4. Suppose that $\Delta_{0}^{\prime}$ is square free and that $\ell \nmid \Delta_{0}^{\prime}$ is a prime such that

(1) $4 \Delta_{0}^{\prime} \ell$ is a fundamental discriminant and $\Delta_{0}^{\prime} \ell \equiv 7(\bmod 8)$;

(2) $\ell>\frac{1}{12}\left(12 H\left(-16 \Delta_{0}^{\prime} \ell\right)+2\right)\left[\Gamma: \Gamma_{0}\left(4 \Delta_{0}^{\prime}\right)\right]$.

Then there are infinitely many odd $m$ for which $c\left(\Delta_{0}^{\prime} \ell m^{2}\right)$ is even.

Remark. For any fixed $\Delta_{0}^{\prime}$ as above, all but finitely many primes $\ell$ with $\Delta_{0}^{\prime} \ell \equiv 7$ $(\bmod 8)$ satisfy the conditions in Theorem 1.4

To obtain these results, we consider the logarithmic derivatives of twisted Borcherds products defined by Zagier [14]. We are then able to interpret the coefficients of $j(z)$ as the coefficients of weight 2 meromorphic modular forms modulo 2 . 
Employing a strategy of Ono [9] using results on nilpotency and the distribution of parity holding for modular forms of integer weight then yields our results.

The paper is organized as follows. In Section 2 we present necessary background information on Borcherds products and the nilpotency of the Hecke algebra. In Section 3 we prove the results about the coefficients of $j(z)$.

\section{Borcherds PRODUCTS AND MODUlAR FORMS MODUlO 2}

2.1. A twisted Borcherds product. In [14] Zagier considers the traces of the modular $j$-invariant. He shows that they are connected to certain basis elements of the space of weakly holomorphic modular forms of weight $1 / 2$ on $\Gamma_{0}(4)$ satisfying the Kohnen plus space condition. More explicitly, for every negative discriminant $-d$, there is a unique (and easily constructable) $f_{d}$ in that space whose Fourier expansion at the cusp $\infty$ is given by

$$
f_{d}(z)=q^{-d}+\sum_{\substack{n \geq 1 \\ n \equiv 0,1(4)}} c_{d}(n) q^{n}
$$

The $f_{d}$ 's form a basis of this space. In particular, $f_{4}$ is given by

$$
f_{4}(z)=f_{0}(z) \cdot j(4 z)-746 \cdot f_{0}(z)-2 \cdot f_{3}(z),
$$

where $f_{0}(z):=\theta(z)=\sum_{n \in \mathbb{Z}} q^{n^{2}}$ is the ordinary Jacobi theta series.

As before we let $\Delta$ be a positive fundamental discriminant which is congruent to 0 modulo 4 .

We then define the function

$$
P_{\Delta}(X):=\prod_{b(\bmod \Delta)}\left(1-e^{2 \pi i b / \Delta} X\right)^{\left(\frac{\Delta}{b}\right)},
$$

where $\left(\frac{\Delta}{b}\right)$ denotes the Kronecker symbol. Then the statement of Theorem 7 of [14] reads as follows (see also Section 8.1 of [2]).

Theorem 2.1. The twisted Borcherds product

$$
\Psi_{\Delta}\left(z, f_{d}\right):=\prod_{m=1}^{\infty} P_{\Delta}\left(q^{m}\right)^{c_{d}\left(\Delta m^{2}\right)}
$$

is a meromorphic modular form of weight 0 for the group $S L_{2}(\mathbb{Z})$ whose divisor on $X(1)$ is supported on $C M$ points of discriminant $-\Delta d$.

Using this result we can easily obtain Theorem 1.1

Proof of Theorem 1.1. We let $\Theta:=q \frac{d}{d q}$. Then $\Theta\left(\Psi_{\Delta}\left(z, f_{d}\right)\right)$ is a weight 2 meromorphic modular form on $\Gamma$. Therefore, the logarithmic derivative $\frac{\Theta\left(\Psi_{\Delta}\left(z, f_{d}\right)\right)}{\Psi_{\Delta}\left(z, f_{d}\right)}$ is a weight 2 meromorphic modular form on $\Gamma$ whose poles are simple and are supported at the $\Gamma$-CM points of discriminant $-\Delta d$.

Using (2.3) it is not hard to show that

$$
\frac{\Theta\left(P_{\Delta}\left(q^{m}\right)\right)}{P_{\Delta}\left(q^{m}\right)}=-\sqrt{\Delta} m \sum_{n=1}^{\infty}\left(\frac{\Delta}{n}\right) q^{n m} .
$$

We now let $d=4$. For the logarithmic derivative of $\Psi_{\Delta}(z)$ we obtain

$$
\mathfrak{F}(\Delta ; z):=-\frac{1}{\sqrt{\Delta}} \frac{\Theta\left(\Psi_{\Delta}(z)\right)}{\Psi_{\Delta}(z)}=\sum_{m=1}^{\infty} m c_{4}\left(\Delta m^{2}\right) \sum_{n=1}^{\infty}\left(\frac{\Delta}{n}\right) q^{n m} .
$$


Since, by (2.2), $f_{4}(z) \equiv j(4 z)(\bmod 2)$ we have that $c_{4}(4 m) \equiv c(m)(\bmod 2)$, which implies

$$
c_{4}\left(\Delta m^{2}\right) \equiv c\left(\Delta^{\prime} m^{2}\right) \quad(\bmod 2) .
$$

Combined with the fact that $\left(\frac{\Delta}{n}\right)=0$ if $\operatorname{gcd}(n, \Delta) \neq 1$, we obtain

$$
\mathfrak{F}(\Delta ; z) \equiv \sum_{\substack{m=1 \\ \operatorname{gcd}(m, 2)=1}}^{\infty} c\left(\Delta^{\prime} m^{2}\right) \sum_{\substack{n=1 \\ \operatorname{gcd}(n, \Delta)=1}}^{\infty} q^{n m} \quad(\bmod 2) .
$$

2.2. Local nilpotency and the distribution of odd coefficients. Here we recall several results about the nilpotency of the Hecke algebra on modular forms modulo 2 and the distribution of odd coefficients. All of these can be found in 8 ] and [9].

Let $f(z)$ be an integer weight modular form on $\Gamma_{0}(N)$ with integer coefficients. If $f(z) \not \equiv 0(\bmod 2)$, then we say that $f(z)$ has degree of nilpotency $i$ if there are primes $p_{1}, p_{2}, \ldots, p_{i-1}$ not dividing $2 N$ for which

$$
f(z)\left|T_{p_{1}}\right| T_{p_{2}}|\cdots| T_{p_{i-1}} \not \equiv 0 \quad(\bmod 2),
$$

and if for every set of primes $\ell_{1}, \ell_{2}, \ldots, \ell_{i}$ not dividing $2 N$ we have

$$
f(z)\left|T_{\ell_{1}}\right| T_{\ell_{2}}|\cdots| T_{\ell_{i}} \equiv 0 \quad(\bmod 2) .
$$

It is known that the action of Hecke algebras on spaces of modular forms with integer coefficients is locally nilpotent modulo 2. In particular, this result was proven by Tate [13] for modular forms of integer weight on $\mathrm{SL}_{2}(\mathbb{Z})$. His result was later generalized by Ono and Taguchi [10]. Moreover, Ono proved the following results.

Lemma 2.2 (Lemma 3.2 of [9]). Suppose that $f(z)=\sum_{n=1}^{\infty} a(n) q^{n} \in S_{k}\left(\Gamma_{0}(N)\right.$ ) with integer coefficients has degree of nilpotency $i>0$. Then the following are true:

(1) There are primes $p_{1}, p_{2}, \ldots, p_{i-1}$ not dividing $2 N$, and an integer $n_{0}$ such that

$$
a\left(n_{0} M^{2} p_{1} p_{2} \cdots p_{i-1}\right) \equiv 1 \quad(\bmod 2)
$$

for every integer $M \geq 1$ that is coprime to $2 p_{1} p_{2} \cdots p_{i-1} N$.

(2) If $\ell_{1}, \ell_{2}, \ldots, \ell_{i}$ are primes not dividing $2 N$, then

$$
a\left(n \ell_{1} \ell_{2} \cdots \ell_{i}\right) \equiv 0 \quad(\bmod 2)
$$

for every $n$ coprime to $\ell_{1}, \ell_{2}, \ldots, \ell_{i}$.

Theorem 2.3 (Theorem 3.3 of [9]). If $f(z)=\sum_{n=1}^{\infty} a(n) q^{n} \in S_{k}\left(\Gamma_{0}(N)\right)$ has integer coefficients, then the following are true:

(1) A positive proportion of the primes $p \equiv-1(\bmod 2 N)$ have the property that

$$
f(z) \mid T_{p} \equiv 0 \quad(\bmod 2)
$$

(2) Suppose that $n_{0}$ is an integer coprime to $N$ with the property that

$$
a\left(n_{0} p_{1} p_{2} \cdots p_{i-1}\right) \equiv 1 \quad(\bmod 2),
$$

where $p_{1}, p_{2}, \ldots, p_{i-1}$ are primes which do not divide $2 n_{0} N$. If $M$ is an integer coprime to $2 N$ and $\operatorname{gcd}(r, M)=1$, then

$\#\left\{m \leq X: a\left(n_{0} m\right) \equiv 1(\bmod 2)\right.$ and $\left.m \equiv r(\bmod M)\right\} \gg \frac{X}{\log X}(\log \log X)^{i-2}$. 


\section{Proof of Theorems 1.2, 1.3, and 1.4}

Here we prove Theorems 1.2, 1.3, and 1.4, where we argue analogously to Ono in 9 .

We let $H_{D}(X)$ be the usual Hilbert class polynomial of discriminant $-D$.

Lemma 3.1. There is a holomorphic modular form of weight $12 H(-4 \Delta)+2$ on $\Gamma$ with integer coefficients which is congruent to

$$
\tilde{J}(\Delta ; z) H_{4 \Delta}(j(z)) \Delta(z)^{H(-4 \Delta)} \quad(\bmod 2) .
$$

Proof. Let $\mathfrak{F}(\Delta ; z)$ be as in (2.4). Then $\mathfrak{F}(\Delta ; z)$ is a meromorphic modular form with integer coefficients which is congruent to $\tilde{J}(\Delta ; z)$ modulo 2 . Moreover, its poles are simple and are supported on $\mathrm{SL}_{2}(\mathbb{Z})-\mathrm{CM}$ points of discriminant $-4 \Delta$. Since $H_{4 \Delta}(j(z))$ has a zero of the same order at each such CM point and $\Delta(z)^{H(-4 \Delta)}$ accounts for the poles at $\infty$ introduced by $H_{4 \Delta}(j(z))$, the claim follows.

Proof of Theorem 1.3. We first prove (1). Assume that all $c\left(\Delta^{\prime} m^{2}\right)$ for odd $m$ are odd, which implies that

$$
\tilde{J}(\Delta ; z) \equiv \sum_{\substack{m=1 \\ \operatorname{gcd}(m, 2)=1}}^{\infty} \sum_{\substack{n=1 \\ \operatorname{gcd}(n, \Delta)=1}}^{\infty} q^{m n}(\bmod 2) .
$$

We also have the classical identity

$$
\Omega(z):=\frac{\eta(4 z)^{8}}{\eta(2 z)^{4}}=\sum_{\substack{n=1 \\ \operatorname{gcd}(n, 2)=1}}^{\infty} \sum_{2 \nmid d \mid n} d q^{n}=q+4 q^{3}+6 q^{5}+\cdots,
$$

where $\eta(z):=q^{1 / 24} \prod_{n=1}^{\infty}\left(1-q^{n}\right)$ is Dedekind's eta-function. It is well known that $\Omega(z)$ is a modular form of weight 2 for $\Gamma_{0}(4)$. It obviously satisfies

$$
\Omega(z) \equiv \sum_{\substack{m=1 \\ \operatorname{gcd}(m, 2)=1}}^{\infty} \sum_{\substack{n=1 \\ \operatorname{gcd}(n, 2)=1}}^{\infty} q^{m n} \quad(\bmod 2) .
$$

Then it is easily seen that

$$
\Omega(\Delta ; z):=\sum_{\substack{1 \leq \delta \mid \Delta^{\prime} \\ \operatorname{gcd}(2, \delta)=1}} \Omega(\delta z) \equiv \sum_{\substack{m=1 \\ \operatorname{gcd}(m, 2)=1}}^{\infty} \sum_{\substack{n=1 \\ \operatorname{gcd}(n, \Delta)=1}}^{\infty} q^{m n}(\bmod 2)
$$

In particular, we have that $\tilde{J}(\Delta ; z) \equiv \Omega(\Delta ; z)(\bmod 2)$. By combining the fact that $\Omega(\Delta ; z)$ is a modular form of weight 2 on $\Gamma_{0}\left(4 \Delta^{\prime}\right)$ with Lemma 3.1, it follows that

$$
\tilde{J}(\Delta ; z) H_{4 \Delta}(j(z)) \Delta(z)^{H(-4 \Delta)} \equiv \Omega(\Delta ; z) H_{4 \Delta}(j(z)) \Delta(z)^{H(-4 \Delta)} \quad(\bmod 2)
$$

in $M_{12 H(-4 \Delta)+2}\left(\Gamma_{0}\left(4 \Delta^{\prime}\right)\right)$. Sturm's theorem [12] now implies that this holds if and only if the first $\frac{12 H(-4 \Delta)+2}{12}\left[\Gamma: \Gamma_{0}\left(4 \Delta^{\prime}\right)\right]$ coefficients are congruent modulo 2 . This proves the bound concerning the first (if any) even value. 
Now assume there are only finitely many odd $m$ for which $c\left(\Delta^{\prime} m^{2}\right)$ is even. Denote these $m$ by $m_{1}, m_{2}, \ldots, m_{s}$ and let $m_{1}$ be the smallest of these numbers. Using (3.2) we see that

$$
\Omega(\Delta ; z)-\tilde{J}(\Delta ; z) \equiv \sum_{i=1}^{s} \sum_{\substack{n=1 \\ \operatorname{gcd}(n, \Delta)=1}}^{\infty} q^{m_{i} n} \quad(\bmod 2) .
$$

We now define $T(\Delta ; z)$ to be the level 1 cusp form

$$
T(\Delta ; z):=\sum_{n=5}^{\infty} t(\Delta ; n) q^{n}:=H_{4 \Delta}(j(z)) \Delta(z)^{H(-4 \Delta)+5}=q^{5}+\cdots
$$

Then the Hecke algebra acts locally nilpotently on $T(\Delta ; z)$ (see Section 2.2). The degree of nilpotency is an integer $a \geq 2$ since the first coefficient of $T(\Delta ; z)$ is odd. Theorem 2.3 (2) now implies that in every arithmetic progression $r(\bmod M)$, where $M$ is an odd integer coprime to $r$, that

$$
\#\{p \leq X: t(\Delta ; p) \equiv 1 \quad(\bmod 2) \text { and } p \equiv r \quad(\bmod M)\} \gg X / \log X .
$$

Combining the properties of $\Omega(\Delta ; z)$ and Lemma 3.1 yields the existence of a cusp form $S(\Delta ; z)$ on $\Gamma_{0}\left(4 \Delta^{\prime}\right)$ for which

$$
S(\Delta ; z)=\sum_{n=1}^{\infty} s(\Delta ; n) q^{n} \equiv(\Omega(\Delta ; z)-\tilde{J}(\Delta ; z)) T(\Delta ; z) \quad(\bmod 2) .
$$

For a positive integer $N$ we find by using equation (3.4) that

$$
s(\Delta ; N) \equiv \sum_{i=1}^{s} \sum_{\substack{n=1 \\ \operatorname{gcd}(n, \Delta)=1}}^{\infty} t\left(\Delta ; N-m_{i} n\right) \quad(\bmod 2) .
$$

These sums are finite because $t(\Delta ; k)=0$ for $k \leq 5$.

Theorem 2.3 (1) implies that there are infinitely many primes $p$ for which

$$
s(\Delta ; n) \equiv 0 \quad(\bmod 2)
$$

when $p \| n$. Let $p_{0} \nmid m_{1} m_{2} \cdots m_{s} \Delta$ be such a prime and let $Y:=p_{0}^{2} \Delta \operatorname{lcm}\left(m_{1}, \ldots\right.$, $m_{s}$ ). Our construction of $S(\Delta ; z)$ together with (3.7) implies that

$$
s(\Delta ; N+Y)-s(\Delta ; N) \equiv \sum_{i=1}^{s} \sum_{\substack{1 \leq n \leq \frac{Y}{m_{i}} \\ \operatorname{gcd}(n, \Delta)=1}} t\left(\Delta ; N+Y-m_{i} n\right) \quad(\bmod 2) .
$$

Using this we will construct a contradiction. Consider the arithmetic progression

$$
N \equiv p_{0} \quad\left(\bmod p_{0}^{2}\right) .
$$

For each pair $(i, n)$, where $1 \leq i \leq s$ and $1 \leq n \leq \frac{Y}{m_{i}}$, except $\left(m_{1}, 1\right)$ we consider progressions of the form

$N \equiv-\left(Y-m_{i} n\right)+\ell_{1}(i, n) \ell_{2}(i, n) \cdots \ell_{a}(i, n) \quad\left(\bmod \ell_{1}(i, n)^{2} \ell_{2}(i, n)^{2} \cdots \ell_{a}(i, n)^{2}\right)$.

Choose distinct odd primes $\ell_{j}(i, n)$ which are coprime to $p_{0} m_{1} \cdots m_{s} \Delta$ such that the system in (3.10) and (3.11) has a solution of the form $N \equiv r_{0}\left(\bmod M_{\Delta}\right)$, where $\operatorname{gcd}\left(r_{0}+Y-m_{1}, M_{\Delta}\right)=1$. This solution exists by the Chinese Remainder 
Theorem. By (3.5) there are infinitely many primes $p$ of the form $N_{p}+Y-m_{1}$ for which $t\left(\Delta ; N_{p}+Y-m_{1}\right) \equiv 1(\bmod 2)$. These $p$ satisfy:

(1) We have that $p_{0} \| N_{p}$ and $p_{0} \|\left(N_{p}+Y\right)$ (because $\left.p_{0}^{2} \mid Y\right)$.

(2) For each $(i, n)$ with $1 \leq i \leq s$ and $1 \leq n \leq \frac{Y}{m_{i}}$, except $\left(m_{1}, 1\right)$ we obtain

$$
N_{p}+Y-m_{i} n \equiv \ell_{1}(i, n) \ell_{2}(i, n) \cdots \ell_{a}(i, n) \quad\left(\bmod \ell_{1}(i, n)^{2} \ell_{2}(i, n)^{2} \cdots \ell_{a}(i, n)^{2}\right) .
$$

By (3.8) we now see that $s\left(\Delta ; N_{p}+Y\right)$ and $s\left(\Delta ; N_{p}\right)$ are both even. Lemma 2.2 (2) implies that each summand in (3.9) is even, except $t\left(\Delta ; N_{p}+Y-m_{1}\right)$, since $T(\Delta ; z)$ has degree of nilpotency $a$. But since $t\left(\Delta ; N_{p}+Y-m_{1}\right)$ is odd, we obtain the contradiction $0 \equiv 1(\bmod 2)$ in $(3.9)$. Therefore, there must be infinitely many odd $m$ with the property that $c\left(\Delta^{\prime} m^{2}\right)$ is even unless there is no such $m$.

To prove the second part of the theorem, observe that if $c\left(\Delta^{\prime} m^{2}\right)$ is even for all odd $m$, then $\tilde{J}(\Delta ; z) H_{4 \Delta}(j(z)) \Delta(z)^{H(-4 \Delta)}$ is trivial. Together, Lemma (3.1) and Sturm's bound now imply the bound $\frac{1}{12}(12 H(-4 \Delta)+2)$ on the first $m$ (if there are any).

Now suppose that $\tilde{J}(\Delta ; z) \not \equiv 0(\bmod 2)$ but only finitely many $c\left(\Delta^{\prime} m^{2}\right)$ are odd. Then we see that

$$
\tilde{J}(\Delta ; z) \equiv \sum_{i=1}^{s} \sum_{\substack{n=1 \\ \operatorname{gcd}\left(\Delta^{\prime}, n\right)=1}}^{\infty} q^{m_{i} n} \quad(\bmod 2)
$$

and since this is the series in (3.3) the proof of the second part follows mutatis mutandis by replacing $(\Omega(\Delta ; z)-\tilde{J}(\Delta ; z)) T(\Delta ; z)$ with $\tilde{J}(\Delta ; z) T(\Delta ; z)$ in (3.6).

Proof of Theorem 1.4. Assume on the contrary that $c\left(\Delta_{0}^{\prime} \ell m^{2}\right)$ is odd for every odd $m$. As before we see that

$$
\tilde{J}\left(\Delta_{0} \ell ; z\right) \equiv \Omega\left(\Delta_{0} \ell ; z\right)=\sum_{\substack{1 \leq \delta \mid \Delta_{0}^{\prime} \ell \\ \operatorname{gcd}(2, \delta)=1}} \Omega(\delta z) \quad(\bmod 2),
$$

where $\Delta_{0}=4 \Delta_{0}^{\prime}$. The fact that $\Omega(\delta z)$ is a holomorphic modular form of weight 2 on $\Gamma_{0}(4 \delta)$ together with Lemma 3.1 implies that

$$
\sum_{n=1}^{\infty} a(n) q^{n}:=\left(\tilde{J}\left(\Delta_{0} \ell ; z\right)-\sum_{\substack{1 \leq \delta \mid \Delta_{0}^{\prime} \\ \operatorname{gcd}(2, \delta)=1}} \Omega(\delta z)\right) H_{4 \Delta_{0} \ell}(j(z)) \Delta(z)^{H\left(-4 \Delta_{0} \ell\right)}
$$

is congruent to a weight $2+12 H\left(-4 \Delta_{0} \ell\right)$ holomorphic modular form on $\Gamma_{0}\left(4 \Delta_{0}^{\prime}\right)$ modulo 2. Using (3.12) we see that

$$
\sum_{n=1}^{\infty} a(n) q^{n} \equiv H_{4 \Delta_{0} \ell}(j(z)) \Delta(z)^{H\left(-4 \Delta_{0} \ell\right)} \sum_{\substack{1 \leq \delta \mid \Delta_{0}^{\prime} \\ \operatorname{gcd}(2, \delta)=1}} \Omega(\delta \ell z) \equiv q^{\ell}+\cdots \quad(\bmod 2) .
$$

This contradicts Sturm's bound [12] for the first odd coefficient, which implies that

$$
\ell \leq \frac{1}{12}\left(12 H\left(-16 \Delta_{0}^{\prime} \ell\right)+2\right)\left[\Gamma: \Gamma_{0}\left(4 \Delta_{0}^{\prime}\right)\right] .
$$

Proof of Theorem 1.2. We let $\Delta_{0}^{\prime}:=1$ in Theorem 1.4. If $\ell \equiv 7(\bmod 8)$ is a prime for which

$$
\ell>6 H(-16 \ell)+1
$$


then the claim follows from Theorem 1.4 Dirichlet's class number formula implies that, for the classical class number,

$$
h(-\ell)<\frac{1}{\pi} \sqrt{\ell} \log (\ell) .
$$

Since here $H(-16 \ell)=4 h(-\ell)$ (see for example page 273 of [3] ), (3.13) holds for all $\ell \geq 4023$. The theorem follows from applying Theorem 1.3 (1) to each prime $\ell<4023$.

\section{ACKNOWLEDGEMENTS}

The author thanks Ken Ono for valuable discussions and his guidance throughout the project. Moreover, the author thanks Stephan Ehlen, Marie Jameson, Eric Larson, Robert Lemke Oliver, and Larry Rolen for helpful discussions and comments.

\section{REFERENCES}

[1] S. Ahlgren and K. Ono, Arithmetic of singular moduli and class polynomials, Compositio Mathematica 141 (2005), pages 293-312. MR2134268 (2006a:11058)

[2] J. H. Bruinier and K. Ono, Heegner divisors, L-functions and harmonic weak Maass forms, Annals of Mathematics 172 (2010), pages 2135-2181. MR2726107

[3] H. Cohen, Sums involving the values at negative integers of L-functions of quadratic characters, Math. Ann. 217 (1975), pages 271-285. MR0382192 (52:3080)

[4] O. Kolberg, Congruences for the coefficients of the modular invariant $j(\tau)$, Math. Scand. 10 (1962), pages 173-181. MR0143735(26:1287)

[5] J. Lehner, Divisibility properties of the Fourier coefficients of the modular invariant $j(\tau)$, Amer. J. Math. 71 (1949), pages 136-148. MR0027801 (10:357a)

[6] J. Lehner, Divisibility properties of the Fourier coefficients of the modular invariant $j(\tau)$, Amer. J. Math. 71 (1949), pages 136-148. MR0027801(10:357a)

[7] J. Lehner, Further congruence properties of the Fourier coefficients of the modular invariant $j(\tau)$, Amer. J. Math. 71 (1949), pages 373-386. MR0027802 (10:357b)

[8] K. Ono, The web of modularity: Arithmetic of the coefficients of modular forms and qseries, CBMS Conference Series 102, Amer. Math. Soc., Providence, RI, 2004. MR2020489 (2005c:11053)

[9] K. Ono, The parity of the partition function, Advances in Mathematics 225 (2010), pages 349-366. MR2669356 (2011m:11209)

[10] K. Ono and Y. Taguchi, 2-adic properties of certain modular forms and their applications to arithmetic functions, International Journal of Number Theory, 1, No. 1 (2005), pages 75-101. MR2172333(2006e:11057)

[11] J.-P. Serre, Divisibilité de certaines fonctions arithmétiques, Enseignement Math. 22 (1976), pages 227-260. MR0434996 (55:7958)

[12] J. Sturm, On the congruence of modular forms, Springer Lect. Notes in Math. 1240, SpringerVerlag, Berlin, 1987, pages 275-280. MR894516 (88h:11031)

[13] J. Tate, The non-existence of certain Galois extensions of $\mathbb{Q}$ unramified outside 2 , Arithmetic geometry (Tempe, AZ, 1993), Contemp. Math. 174, Amer. Math. Soc., Providence, RI, 1994, pages 153-156. MR.1299740 (95i:11132)

[14] D. Zagier, Traces of singular moduli, Motives, polylogarithms and Hodge theory, Part I, Int. Press Lect. Ser., International Press, 2002, pages 211-244. MR1977587 (2004h:11037)

Fachbereich Mathematik, Technische Universität Darmstadt, Schlossgartenstr. 7 , D-64289 Darmstadt, Germany

E-mail address: alfes@mathematik.tu-darmstadt.de 